\title{
The provision of dental implants in the National Health Service Hospital Dental Services - a national questionnaire
}

\author{
C. J. Butterworth, ' A. M. Baxter, ${ }^{2}$ M. J. Shaw, ${ }^{3}$ and G. Bradnock, ${ }^{4}$
}

\begin{abstract}
Objective To assess the activity of consultants in restorative dentistry in the United Kingdom in the provision of osseointegrated dental implants within the National Health Service Hospital service and to evaluate their attitudes concerning the relevant medical and oral factors considered in patient selection for implant treatment.

Design/Setting Anonymous postal questionnaire in the United Kingdom.

Subjects Consultants in restorative dentistry.

Results Out of the sample of 145,109 consultants (75\%) completed the questionnaire in 1999.54 of the 109 consultants $(49.5 \%)$ are involved in the provision of osseointegrated implant treatment, treating an average of 29 cases/year (range 2-150). However, over one third of the respondents treated 10 or less cases/year. 89\% worked with oral surgeons as an implant team. $68 \%$ used Branemark (Nobel Biocare) implants as their main system. The majority of consultants felt that smoking, psychoses and previous irradiation were the most important medical factors that contra-indicated implant retained restorations whilst untreated periodontitis, poor oral hygiene and uncontrolled caries were the most important oral contra-indications. Many centres were experiencing significant problems with the funding of implant treatment with one centre receiving no funding. The implications for patient care and specialist training are discussed. Conclusions There is a marked variation in the number of patients treated with endosseous dental implants within the United Kingdom National Health Service hospitals. Many consultants treat 10 or fewer patients each year. In the main, there is agreement about the factors that contra-indicate implant treatment; these are in line with national guidelines.
\end{abstract}

$\mathrm{T}$ he use of endosseous dental implants has markedly increased over the past few years. The success rate generally quoted is of the order of $90 \%$ in the mandible and $80 \%$ in the maxilla. ${ }^{1,2}$ Dental implants are successfully used in the restoration of edentate $e^{3-5}$ and partially dentate patients ${ }^{6}$ in addition to single tooth replacement cases. ${ }^{7}$ The use of dental implants in the reconstruction of oral cancer patients is well documented and has significantly improved their rehabilitation. ${ }^{8}$ Large defects of the hard or soft tissues in the maxillofacial region, whether congenital or acquired, are treated more frequently with the aid of dental implants. ${ }^{9}$

${ }^{1}$ Specialist Registrar in Restorative Dentistry, ${ }^{2}$ Specialist Registrar in Restorative Dentistry, ${ }^{3}$ Consultant in Restorative Dentistry, ${ }^{4}$ Senior Lecturer in Dental Public Health, Birmingham Dental Hospital and School, St Chad's Queensway,

Birmingham B4 6NN

${ }^{*}$ Correspondence to: $C$. Butterworth

email:Chris.Butterworth@abscht.wmids.nhs.uk

REFEREED PAPER

Received 12.06.00; Accepted 15.08.00

(C) British Dental Journal 2001; 190: 93-96
The advent of improved bone grafting techniques ${ }^{10}$ and the use of hyperbaric oxygen therapy for patients who have received radiotherapy ${ }^{11}$ has increased the numbers of patients for whom implant placement is possible, providing functional, psychological and aesthetic benefits. ${ }^{12}$

In view of the increased demand for implant treatment in a diverse patient population across a wide spectrum of clinical disorders, the Royal College of Surgeons of England published a guideline in 1997, through the Clinical Audit Committee.

The stated aim was to assist clinical providers and health authorities to make an informed assessment of patients who may be considered suitable for dental implant treatment within the National Health Service (NHS). ${ }^{13}$

This survey was designed to assess the current use of dental implants by consultant grade staff in the specialty of restorative dentistry within the National Health Service. The consultant's criteria for patient selection for dental implants and the general implant casemix of the consultants were also investigated. The questionnaire also invited comments about implant therapy both in their unit and nationally.

\section{Method}

An anonymous postal questionnaire was divided into five sections. The entire questionnaire consisted of twelve questions.

The first section determined clinical special interest of the respondents, whether they undertook implant treatment and the length of service in a consultant post. Only those who provided implant-retained restorations were required to complete the remaining sections of the form.

The second part of the form dealt with the workload generated by implant treatment and included questions concerning implant placement to establish whether the respondents placed implants themselves or worked in a team, with a surgeon performing the implant placement. Questions concerning the implant systems used were asked.

Parts three and four of the survey investigated the factors that are considered relevant when assessing a patient's suitability to receive implants. Consultants were asked to grade each factor as very important, quite important or not at all important.

Part three dealt with general factors including, the patient's age, tobacco smoking habits and any relevant medical history including osteoporosis, diabetes mellitus, previous radiotherapy, infective endocarditis and psychological history.

Section four dealt with the oral factors that need to be considered prior to implant placement. These include mucosal disease, uncontrolled dental caries, untreated periodontal disease, poor oral hygiene and the presence of parafunctional habits.

The final section sought information on the patient groups treated and which groups were treated more commonly. The groups of patients involved were, predominantly, those defined as appropriate for implant treatment in the Royal College of 
Surgeons Guidelines. ${ }^{13}$ These included those suffering from post oro-facial trauma, hypodontia, cleft palate, major jaw resection caused by malignant disease, severe gagging reflex and severe denture intolerance.

Space was left at the end of each section to allow for comment and a specific question was asked at the end of the questionnaire inviting any additional comments.

A draft questionnaire was evaluated using a small group of consultants in restorative dentistry, this resulted in a number of minor revisions being made.

The questionnaires were sent, in a single mailing, to 145 consultants who were all members of the Consultants in Restorative Dentistry Group. Replies were anonymous and returned to the authors in identical pre-paid envelopes enclosed with the questionnaire.

\section{Results}

A total of $109(75.1 \%)$ consultants responded. All questionnaires were completed appropriately. Fifty-four (49.5\%) consultants provided implant-retained restorations within the NHS Hospital services; these consultants had been in post between 2 and 25 years (mean 11.6 years). The total number of consultants and those providing implant treatment compared with the years spent in post is shown in Figure 1.

Sub-specialty interest

Forty-five $(83 \%)$ of respondents who provided dental implants expressed a special interest within the restorative dentistry

\begin{tabular}{lr}
$\begin{array}{l}\text { Table I Monospecialty interests of consultants } \\
\text { surveyed }\end{array}$ \\
\hline Fixed and removable prosthodontics \\
Periodontology & 35 \\
Endodontics & 5 \\
Unspecified & 1 \\
Implantology & 9 \\
Others & 2 \\
\hline
\end{tabular}

specialty. These are shown in Table 1 .

\section{Current implant practice}

Forty-eight $(89 \%)$ worked together with oral surgeons as an implant team. Six (11\%) consultants stated that they placed and restored all implants in all their patients with no reference to an oral

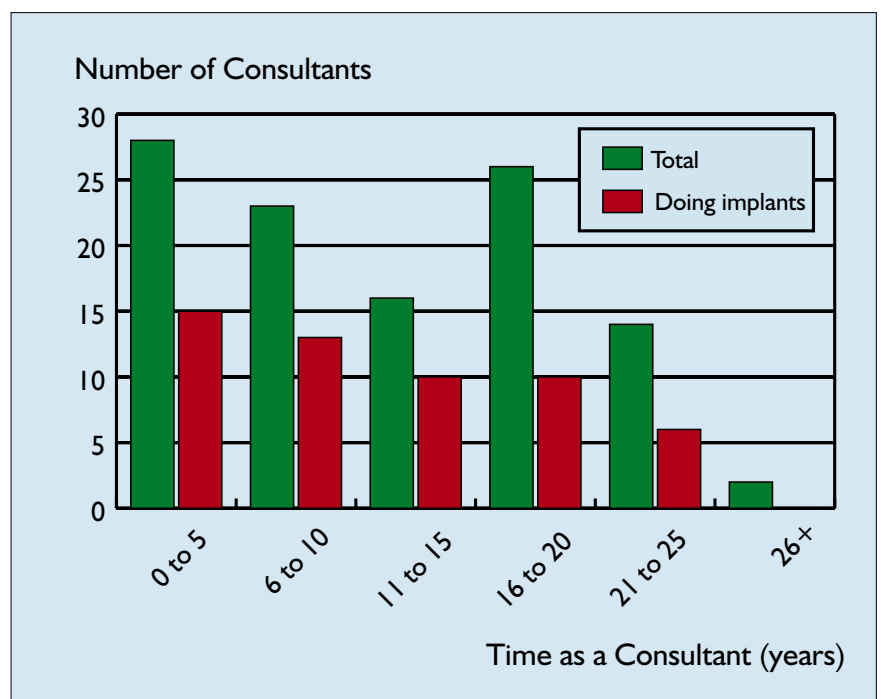

Fig. I The number of consultants providing implant treatment compared with their time in post

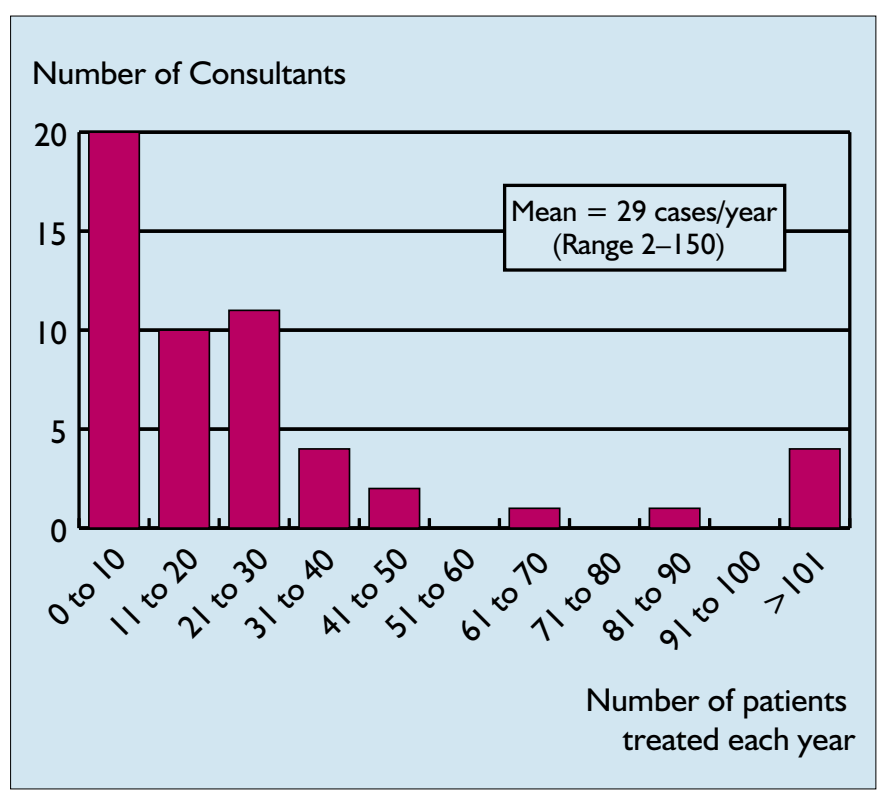

Fig. 2 Number of patients treated with implants each year

surgeon. However, 19 (35\%) consultants placed implants themselves in selected patients in addition to working with an oral surgeon on other cases.

The mean number of patients treated with implants by consultant each year was 29 (range 2-150). The distribution of patients per year is shown in Figure 2. The mean number of sessions each week spent providing implant treatment was 1.7 (range 0.25-8.0).

The most commonly used implant system used was Branemark (Nobel Biocare) with $68 \%$ of consultants stating this as their most frequently used system. Table 2 shows the proportion of consultants and

Table 2 Implant systems commonly used

\begin{tabular}{lc}
\hline Implant system used & \% of consultants \\
\hline & \\
Branemark & 68.5 \\
Straumann & 16.7 \\
Frialit & 5.5 \\
Astra & 3.7 \\
Others & 5.5 \\
\hline
\end{tabular}

the implant systems used as their predominant supplier.

\section{Medical factors influencing patient selection}

Consultants were asked to assess the importance of certain medical factors that need to be considered in patient selection on a 3-point scale; very important, quite important or not important. The data concerning the 'very important' responses are shown in Figure 3.

\section{Oral factors influencing patient selection}

Consultants were asked to grade oral factors that influence patient selection in the same manner to the medical factors. The data concerning the 'very important' responses are shown in Figure 4.

\section{Present casemix}

Consultants were asked to quantify their present casemix from the groups of patients acknowledged as appropriate for implant treatment depending on the numbers they treat from each group. These results are shown in Figure 5.

\section{Comments}

Sixty-eight per cent of comments were related to funding issues. 


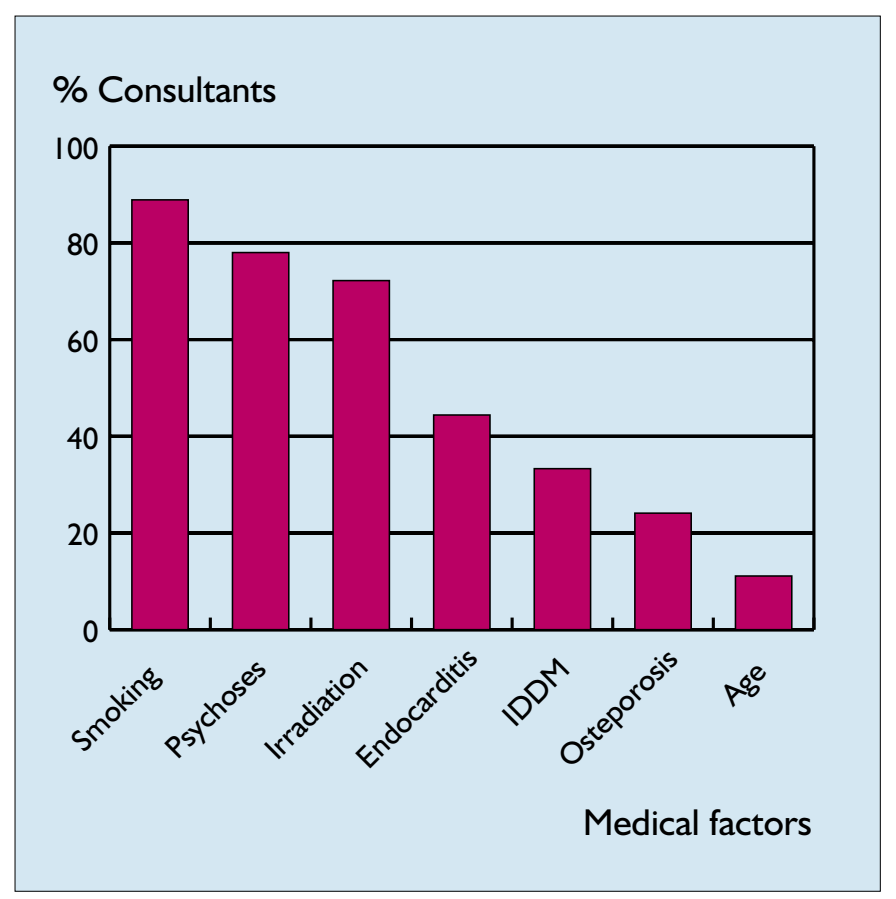

Fig. 3 Medical factors considered very important in patient selection

Other topics raised by respondents were associated with the importance of teamwork and with clinical issues such as the implication of tobacco smoking for patients who might benefit from implant treatment.

\section{Discussion}

This study was designed to identify the amount of implant treatment currently being undertaken within the NHS Hospital Dental services by consultants in restorative dentistry. A good response rate (75\%) from a single mailing bears testimony to the importance attached to this form of treatment by the consultants involved.

A similar study involving a questionnaire, which investigated failure rates of implants, received 39 replies from 120 centres. ${ }^{14}$ Only half of all the consultants who replied were involved in implant treatment but it should be remembered that consultants in restorative dentistry often have monospecialty interests that would not necessarily include implant provision as part of their clinical practice.

The largest group of respondents cited a form of prosthodontics as their mono-specialty interest, an interest that could have been predicted since both fixed and removable restorations increasingly benefit from implant support to replace missing teeth, often to minimise unnecessary tooth destruction. It would appear that the time in post as a consultant is not relevant in the proportion of consultants providing implant treatment.

The majority ( $88 \%$ ) of consultants involved in implant treatment worked with oral surgeons as part of an implant team. This follows the published guideline, ${ }^{13}$ which states 'provider units would be expected to have experienced teams of surgeons, prosthodontists or restorative dentists and suitably trained ancillary staff'. A minority of consultants placed and restored their own implants without this apparent team working. Clearly there are clinical situations when implants can be placed and restored separate from surgical support.

There was a large variation in the number of patients treated by each consultant annually (range 2-150). The Royal College of Surgeons of England guideline also states 'provider units should treat sufficient numbers of patients (with a good case mix) annually to maintain expertise in this demanding area'. There are no published data to indicate what might be considered a 'sufficient' number of cases to maintain such clinical skills.

Certainly the issue of maintaining a high-level of activity has resulted in the ongoing reorganisation of the delivery of care for cleft lip and palate patients in regional centres following the Clinical Standards Advisory Group report. ${ }^{15}$ In many areas of dentistry and medicine a certain level of activity is necessary both to maintain skills and to ensure appropriate future training quality.

Although there is a large number of implants systems on the market currently, the majority of consultants stated their preference for the Branemark system (Nobel-Biocare). Tinsley et al. ${ }^{14}$ also found this system to be the most commonly used in the United Kingdom. This may reflect the substantial research that has been carried out on this system, which to date has the longest follow-up studies of any osseointegrated dental implant system currently on the market. ${ }^{1,16-18}$ However, the wide range of other systems used would indicate that no one system is ideal for every clinical situation.

Attitudes to important issues that feature when treatment planning patients for implant treatment were sought by questioning consultants on their views about relevance of certain social, medical and oral factors considered important within the Royal College of Surgeons of England guideline. There was general agreement on the importance of smoking, psychiatric illness and previous radiotherapy in influencing patient selection for implants. However, the consultants considered that osteoporosis, the effect of previous infective endocarditis and insulin dependent diabetes mellitus as contra-indicators for implant treatment, although important in the selection process, were not as important as the former categories.

Age was rated as the least important of the medical factors although some consultants contributed that young individuals should not receive placement of implants until growth is complete. $^{19}$

Unsurprisingly the presence of untreated periodontitis, uncontrolled caries and poor oral hygiene were considered to be very important contra-indications to patient selection for implant treatment. $^{20}$

Denture intolerance was reported to contribute the largest component of the casemix by about half of the respondents. This may reflect the pattern of referrals to these consultants, many of whom stated that prosthodontics was their monospecialty interest. Only three consultants considered the management of post-cancer care patients reflected the largest proportion of their casemix. There

\section{$\%$ Consultants}

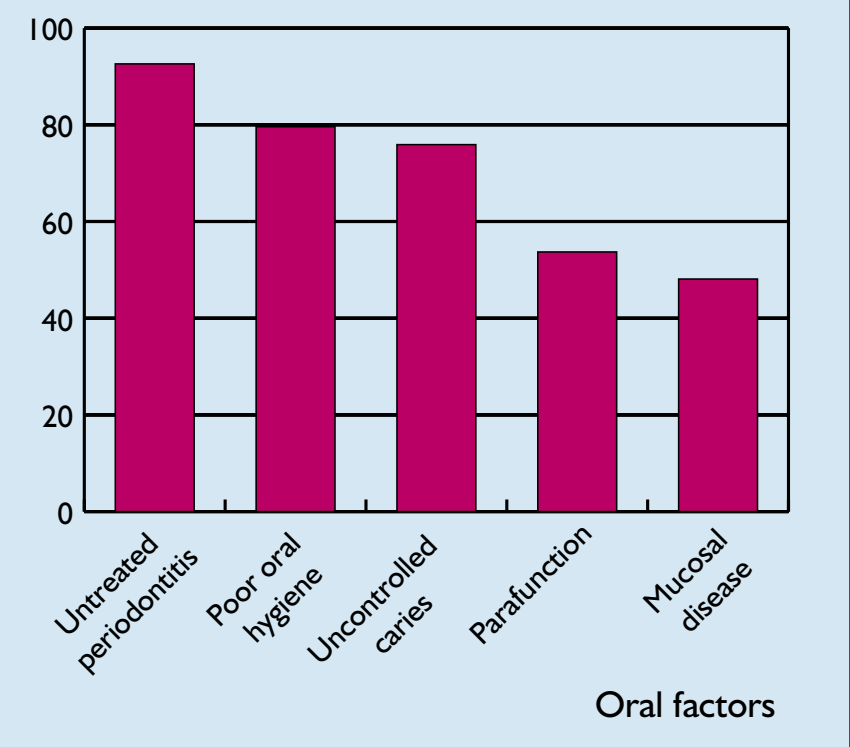

Fig. 4 Oral factors considered very important in patient selection 
Fig. 5 Casemix of patients treated with implants
Number of consultants

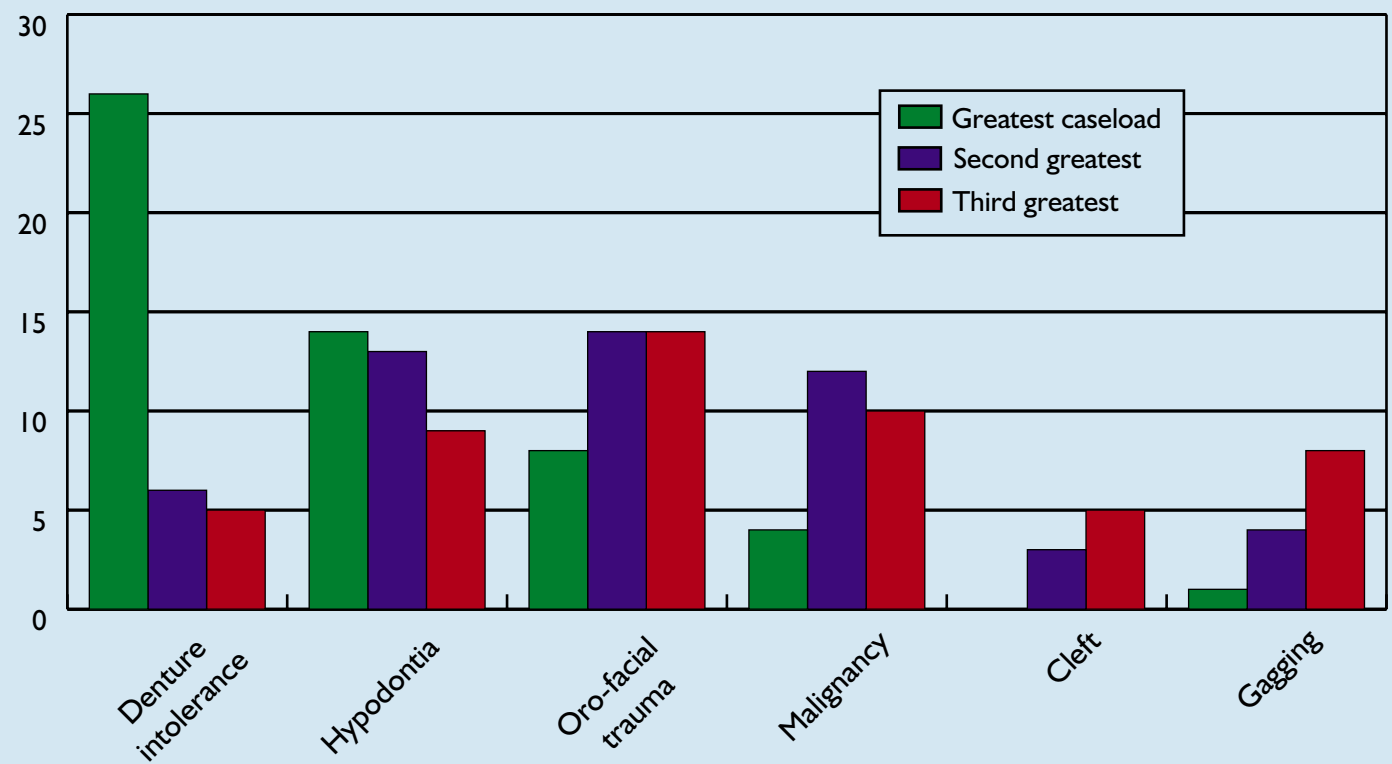

Priority groups continues to be few oncology centres managing head and neck cancer within the United Kingdom that include a restorative dentistry treatment service. The data reflect that lack of service despite the Calman Report emphasis on the essential improvement indicated involving quality of life issues. ${ }^{21}$

Implant treatment is expensive, not only in the initial provision but also in the subsequent maintenance of the implant-retained prosthesis. The majority of comments made confirmed that many centres were experiencing funding difficulties in providing implant treatment.

\section{Conclusions}

This study showed that there is a marked variation in the number of patients treated with dental implants within the UK. There was generalised agreement about the general, medical and oral factors that were important considerations in the selection of patients for implant treatment. Respondents consistently reported funding difficulties in the provision of dental implants in the NHS Hospital Dental Services.

1 Adell R, Eriksson B, Lekholm U. et al. A long-term follow-up study of osseointegrated implants in the totally edentulous jaws. Int J Oral Maxillofac Implants 1990; 5: 347-359.

2 Albrektsson T. A multi-centre report on osseointegrated oral implants. J Pros Dent 1988; 60: 75-84

3 Zarb G A, Schmitt A. Osseointegration and the edentulous predicament. The 10-year Toronto study. Br Dent J 1991; 170: 329-337.

4 Chan M F W-Y, Johnston C, Howell R A, Cawood J I. Prosthetic management of the atrophic mandible using endosseous implants and overdentures: A six year review. Br Dent J 1995; 179: 329-337.

5 Chan M F W-Y, Howell R A, Cawood J I. Prosthetic rehabilitation of the atrophic maxilla using pre-implant surgery and endosseous implants. $\mathrm{Br}$ Dent J 1996; 181: 51-58.

6 Lindh T, Gunne J, Tillberg A et al. A metanalysis of implants in partial edentulism. Clin Oral Implant Res 1998; 9: 80-90.

7 Schmitt A, Zarb G A. The longitudinal clinical effectiveness of osseointegrated dental implants for single tooth replacement. Int J Pros 1993; 6:197-202.

8 Marx R E, Morales M J. The use of implants in the reconstruction of oral cancer patients. Dent Clin N Am 1998; 42: 177-202.

9 Parel S M, Tjellstrom A. The United States and Swedish experience with osseointegration and facial prostheses. Int J Oral Maxillofac Implants 1991; 6: 75-79.

10 Rissolo A R, Bennett J. Bone grafting and its essential role in implant dentistry. Dent Clin N Am 1998; 42: 91-116.

11 Weischer T, Schettler D, Mohr C. Concept of surgical and implant supported prostheses in the rehabilitation of patients with oral cancer. Int J Oral Maxillofac Implants 1996; 11: 775-781.

12 Cune M S, de Putter C, Hoogenstraten J. Treatment outcome with implant retained overdentures: Part II, patient satisfaction and predictability of subjective treatment outcome. J Pros Dent 1994; 72: 152-158.

13 Guidelines for selecting appropriate patients to receive treatment with dental implants: Priorities for the NHS. Faculty of Dental Surgery, National Clinical Guidelines 1997, Royal College of Surgeons.

14 Tinsley D, Watson C J, Ogden A R. A survey of UK centres on implant failures. J Oral Rehab 1999; 26: 14-18.

15 Cleft Lip and Palate. Report of the Clinical Standards Advisory Group. London: HMSO, 1998.

16. Lindquist L W, Carlsson G, Jemt T. A prospective 15-year study of mandibular prostheses supported by osseointegrated implants. Clinical results and marginal bone loss. Clinical Oral Implants Research 1996; 7: 329-336.

17 Henry P J, Laney W R, Jemt, T et al. Osseointegrated implants for single tooth replacement; a retrospective 5-year multi-centre study. Int J Oral Maxfac Implants 1996; 11: 450-455.

18 Lekholm U, van Steenberghe D, Hermann I et al. Osseointegrated implants in the treatment of partially edentulous jaws: a prospective 5-year multicentre study. Int J Oral Maxfac Implants 1994; 9: 627-635.

19 Westwood R M, Duncan J M. Implants in adolescents: a literature review and case reports. Int J Oral Maxillofac Implants 1996; 11: 750-5.

20 Lekholm U. The Surgical Site. In Lindhe J, Karring K and Lang N P (eds) Clinical Periodontology and Implant Dentistry. 3rd ed. pp 890-905. Copenhagen: Munksgaard, 1998.

21 Calman K, Hine D. A Policy Framework for Commissioning Cancer Services: A report by the expert advisory group on cancer to the Chief Medical Officers of England and Wales. Department of Health, 1995. 